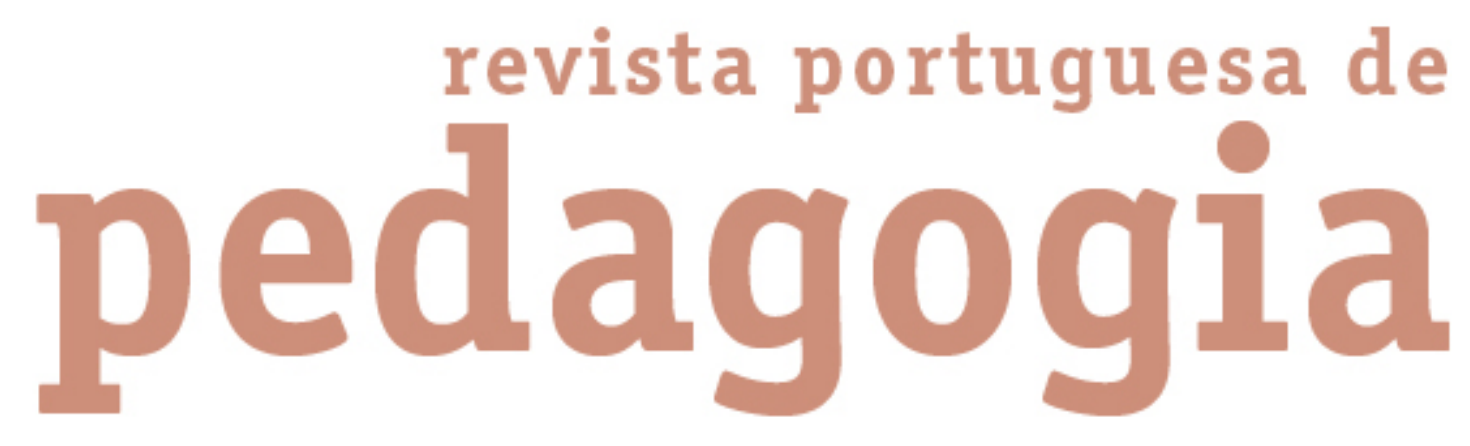

O papel dos mecanismos de aprendizagem implícitos e explícitos na aquisição de uma segunda língua: implicações pedagógicas
Autor(es):
Castro, Catarina

Publicado por: Imprensa da Universidade de Coimbra

URL

persistente:

URI:http://hdl.handle.net/10316.2/38593

DOI:

DOI:http://dx.doi.org/10.14195/1647-8614_49-2_1

Accessed : $\quad$ 26-Apr-2023 16:31:06

A navegação consulta e descarregamento dos títulos inseridos nas Bibliotecas Digitais UC Digitalis, UC Pombalina e UC Impactum, pressupõem a aceitação plena e sem reservas dos Termos e Condições de Uso destas Bibliotecas Digitais, disponíveis em https://digitalis.uc.pt/pt-pt/termos.

Conforme exposto nos referidos Termos e Condições de Uso, o descarregamento de títulos de acesso restrito requer uma licença válida de autorização devendo o utilizador aceder ao(s) documento(s) a partir de um endereço de IP da instituição detentora da supramencionada licença.

Ao utilizador é apenas permitido o descarregamento para uso pessoal, pelo que o emprego do(s) título(s) descarregado(s) para outro fim, designadamente comercial, carece de autorização do respetivo autor ou editor da obra.

$\mathrm{Na}$ medida em que todas as obras da UC Digitalis se encontram protegidas pelo Código do Direito de Autor e Direitos Conexos e demais legislação aplicável, toda a cópia, parcial ou total, deste documento, nos casos em que é legalmente admitida, deverá conter ou fazer-se acompanhar por este aviso. 


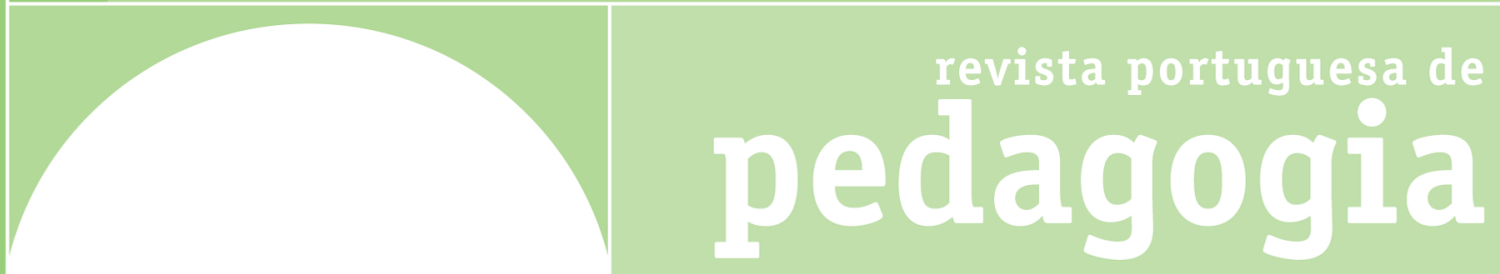




\title{
0 Papel dos Mecanismos de Aprendizagem Implícitos e Explícitos na Aquisição de uma Segunda Língua: Implicações Pedagógicas
}

\author{
Catarina Castro'
}

\begin{abstract}
Resumo
O artigo confronta diferentes posições que têm sido sustentadas na área da Aquisição de Segundas Línguas em relação ao modo como a instrução pode facilitar a aquisição de um novo idioma e, considerando que, em particular, os estudantes adultos beneficiam tanto com o desenvolvimento de conhecimento implícito como explícito para serem capazes de usar a língua-alvo em contextos reais de comunicação, sugere, com base em várias investigações desenvolvidas na área, que o Ensino de Línguas Baseado em Tarefas pode ser uma abordagem pedagógica adequada para promover o tipo de processo de aprendizagem que se considera facilitar a aquisição de um novo idioma.
\end{abstract}

Palavras-chave: aquisição de segundas línguas; instrução e aquisição de L2; ensino de línguas baseado em tarefas

\section{The Role of Implicit and Explicit Learning Processes in Second Language Acquisition: Pedagogical Implications}

\begin{abstract}
In terms of Second Language Acquisition research there are different perspectives on how instruction can best facilitate language acquisition. The article seeks to confront these different positions and, considering that, in particular, adult learners benefit with the development of both implicit and explicit knowledge to be able to use the target language in real-world contexts, suggests that, according to research, Task-Based Language Teaching may be an appropriate pedagogical approach to promote the kind of learning process that is considered to facilitate second language acquisition.
\end{abstract}

1 Fundação para a Ciência e Tecnologia/Faculdade de Ciências Sociais e Humanas de Lisboa. Email: castro.aclm@googlemail.com 
Keywords: second language acquisition; instruction and L2 acquisition; task-based language teaching

\title{
El Papel de los Mecanismos de Aprendizaje Implícitos y Explícitos en la Adquisición de una Segunda Lengua: Implicaciones Pedagógicas
}

\begin{abstract}
Resumen
El artículo compara diferentes posiciones que han emergido en el área de la Adquisición de Segundas Lenguas sobre la manera como la instrucción puede facilitar la adquisición de una nueva lengua y, considerando que, en particular, los estudiantes adultos se benefician tanto con el desarrollo de conocimiento explícito como implícito para usar el idioma de aprendizaje en contextos reales de comunicación, sugiere, con base en algunas investigaciones desarrolladas en el área, que la Enseñanza de Lenguas Basada en Tareas puede ser un enfoque pedagógico apropiado para promover el tipo de proceso de aprendizaje que se considera facilitar la adquisición de un nuevo idioma.
\end{abstract}

Palabras clave: adquisición de segundas lenguas; instrucción y adquisición de L2; enseñanza de lenguas basada en tareas

\section{Introdução}

A Aquisição de Segundas Línguas (ASL), enquanto subdisciplina da Linguística Aplicada, é ainda um campo de estudo recente, no âmbito do qual é possível encontrar posições diferentes em relação ao papel desempenhado pela instrução no processo de aquisição de segundas línguas (L2) ${ }^{2}$, questionando-se, em particular, se a instrução deve adotar uma abordagem tradicional com "foco nas formas" (isto é, envolvendo o ensino sistemático de características gramaticais, com base em um programa estrutural) ${ }^{3}$, ou, pelo contrário, se deve recorrer a uma abordagem com "foco na forma" (ou seja, promovendo a atenção acidental para características da língua-alvo durante a atividade comunicativa, com base em um programa baseado em tarefas), ou, até, basear-se em uma combinação das duas abordagens. Destaca-se, ainda, a

2 Sem descurar as diferenças entre a aquisição precoce e tardia, o conceito de segunda língua (L2) será usado, genericamente, para referir qualquer outra língua que o indivíduo tenha adquirido depois da língua materna.

3 Programas baseados em aspetos gramaticais e estruturais (como verbos, orações, preposições). 
existência de entendimentos diferentes relativamente ao papel desempenhado pelo conhecimento explícito no desenvolvimento da competência comunicativa, o que no seu conjunto reflete tanto a complexidade do objeto de estudo como o caráter ainda jovem da área de investigação.

Partindo, assim, do princípio de que a instrução pode facilitar a aquisição de L2 e considerando também que, em particular, os estudantes adultos beneficiam tanto com o desenvolvimento de conhecimento implícito como explícito ${ }^{4}$ para serem capazes de usar a língua-alvo em contextos reais de comunicação, no presente artigo iremos analisar de que modo o ensino formal pode maximizar este processo, destacando, em termos de abordagens pedagógicas, o Ensino de Línguas Baseado em Tarefas, que, de acordo com alguns estudos longitudinais (e.g., Beretta \& Davies, 1985; De la Fuente, 2006; R. Ellis, Tanaka, \& Yamazaki, 1994; Mackey, 1999; Prabhu, 1987; Shintani, 2013), é globalmente melhor e cria contextos mais propícios à aquisição do que a metodologia baseada na apresentação, prática e produção (R. Ellis \& Shintani, 2014, p. 158), que atualmente tende a ser predominantemente adotada nos cursos de línguas.

\section{0 papel da instrução na aquisição de L2}

Antes de abordarmos o papel desempenhado pela instrução no desenvolvimento da competência comunicativa, começamos por clarificar o sentido que iremos atribuir a dois conceitos fundamentais - o de aquisição e aprendizagem - que têm sido, frequentemente, usados como sinónimos, embora autores como Krashen (1982, 1985,2003 ) insistam em estabelecer distinções significativas entre os dois, usando "aprendizagem" para referir a acumulação de conhecimento metalinguístico e declarativo sobre L2, e "aquisição" para descrever o ganho de conhecimento implícito que resulta na capacidade de usar a língua para comunicar. Não obstante, e embora consideremos importante distinguir os dois processos, no presente artigo optaremos por usar os conceitos "conhecimento explícito versus implícito" ou "conhecimento declarativo versus procedimental"5 para estabelecer esta distinção, sendo os termos "aquisição" e "aprendizagem" usados indistintamente.

4 De acordo com a "posição de interface moderada" em que nos baseamos, no artigo sustentamos que o conhecimento explícito é importante porque cria condições favoráveis ao desenvolvimento do conhecimento implícito.

5 A diferença entre o conhecimento explícito e implícito, por um lado, e o conhecimento declarativo e procedimental, por outro, diz respeito à relação que alguns autores propõem existir (ou não) entre os dois tipos de conhecimento. Assim, enquanto o conhecimento explícito e implícito são vistos como modulares, 
Quanto ao papel facilitador, que é geralmente atribuído à instrução no processo de aquisição de L2, consideramos importante começar por sublinhar que, ao contrário do que muitos professores e estudantes tendem a assumir, esta não é uma questão consensual, como conclui Ortega (2007, pp. 240-242) que, ao analisar algumas das principais teorias que têm emergido na área da ASL, identifica três posições distintas sobre o papel que atribuem à instrução no processo de aquisição de $L 2$, enunciando as que não atribuem qualquer efeito, ${ }^{6}$ as que consideram que tem apenas um efeito limitado ${ }^{7}$, bem como as que sustentam que a instrução pode ter efeitos benéficos ${ }^{8}$ (Ortega, p. 242).

Assim, e embora não seja uma questão consensual, existem, de facto, algumas propostas teóricas de acordo com as quais a instrução desempenha um papel importante quando facilita os processos naturais de aquisição de $L 2$, sendo também de assinalar vários trabalhos (e.g., Housen \& Pierrard, 2005; Long, 1983; Mackey \& Goo, 2007; Norris \& Ortega, 2000; Shintani, Li, \& R. Ellis, 2013; Spada \& Tomita, 2010), que apresentam evidências de que a instrução pode ser importante para a aquisição de L2, podendo mesmo ser necessária para a aquisição de níveis elevados de proficiência, em particular por estudantes adultos.

Verificamos, portanto, que apesar de os investigadores (e.g., R. Ellis, 2005; Howard, 2005; Krashen, 2003) assumirem que as fases de desenvolvimento e os processos cognitivos são os mesmos, tanto na aquisição que ocorre em contexto formal de aprendizagem como em contextos de aprendizagem naturalística (ou seja, em contexto de imersão sem instrução), alguns autores tendem a considerar que a instrução pode contribuir para uma progressão mais rápida dos estudantes, assim como para evitar a fossilização?.

A aquisição de uma nova língua é, sem dúvida, um processo bastante complexo que decorre da influência de inúmeros fatores, como as condições de aprendizagem, especificidades da língua de aprendizagem, fatores sociais, culturais e económicos,

\footnotetext{
o que significa que são armazenados em partes diferentes do cérebro, o conhecimento declarativo e procedimental são frequentemente abordados (Dekeyser, 2007a) como um contínuo, no qual existe lugar para o conhecimento declarativo procedimentalizado e automatizado pela prática.

6 Na primeira categoria, Ortega refere a Teoria da Gramática Universal, a Teoria da Processabilidade, a designada Autonomous Induction Theory e o Concept-Oriented Approach.

7 Na segunda categoria, Ortega destaca a Teoria Sociocultural ( Lantolf \& Thorne, 2007; Vygotsky, 1962, 1978) e algumas Teorias Conexionistas (N. Ellis, 2007).

8 Na terceira categoria, Ortega destaca a Abordagem Interacionista, a Teoria da Aquisição de Capacidades e o Processamento de input.

9 O conceito de fossilização tem sido definido como a cessação da aprendizagem, isto é, a não ocorrência de mudanças na interlíngua do estudante de L2 (Gass \& Selinker, 2008, p. 517).
} 
que afetam não só o estatuto das línguas como o do próprio estudante, mas também de características individuais, como a idade, a aptidão linguística ou a motivação.

No que diz respeito à idade de exposição a $L 2$, e apesar de não haver consenso quanto à existência de um período crítico ou à possibilidade de os estudantes poderem atingir um nível de competência equiparável à de um falante nativo, "[...] there is some evidence for an age-related decline in abilities" (Gass \& Selinker, 2008, p. 414), havendo dados que apontam para o facto de os estudantes mais jovens (sobretudo as crianças) revelarem vantagem na aquisição da fonologia e maior probabilidade de atingirem um nível de proficiência próxima de um falante nativo, embora sejam também reconhecidas algumas vantagens aos estudantes adultos, nomeadamente, em relação ao ritmo de aprendizagem da sintaxe e da morfologia, concluindo-se que: "[t]here is general consensus that most older individuals cannot reasonably hope to ever achieve a native accent in a second language. There is no such consensus about other areas of language" (Gass \& Selinker, 2008, p. 407).

A este respeito, Ullman (2001, 2004) e Paradis (2004, 2009) destacam o papel central dos subsistemas de memória (a declarativa ou explícita e a procedimental ou implícita) na aprendizagem de uma língua (nativa ou segunda), ainda que a capacidade para aprendermos através destes subsistemas de memória se vá alterando ao longo da vida (Ullman, 2001), sendo que a capacidade para aprendermos uma segunda língua por via da memória procedimental/implícita diminui com a idade:

[...] quanto mais avançada a idade, mais o indivíduo recorre à sua memória declarativa para aprender uma $L 2$, ou seja, o aprendente tardio de uma segunda língua recorre a formas de aprendizagem diferentes das que estão ao alcance do indivíduo no momento de aquisição da sua língua materna. As diferenças são particularmente expressivas na aprendizagem da morfossintaxe e da fonologia da L2, pois são sobretudo estes os domínios linguísticos que a criança assimila inconscientemente através da sua memória procedimental. O aprendente tardio, por sua vez, terá que os aprender explicitamente, mobilizando a memória declarativa. Esta forma de aprendizagem exige muita atenção e repetição contínua da estrutura a assimilar, não garantido um sucesso imediato. (Flores, 2013, p. 8)

Ullman (2004, p. 256) defende, no entanto, que o conhecimento linguístico armazenado na memória declarativa do aprendente tardio pode passar para o subsistema da memória procedimental, tornando-se assim implícito e inconsciente, se o falante estiver intensivamente exposto à sua $L 2$, por exemplo através da imersão. 
Como veremos na secção seguinte, esta não é uma posição consensual, sendo que o papel desempenhado pelo conhecimento declarativo (ou explícito) na aquisição de L2 tem sido um tópico bastante controverso, que tem dado origem a posições muito diferentes sobre a relação entre os dois tipos de conhecimento (implícito/ procedimental e explícito/declarativo), que ficou conhecida como Hipótese de interface, a que se encontram associadas propostas pedagógicas também distintas que continuam até hoje a ser debatidas.

\section{0 desenvolvimento da competência comunicativa}

Os professores e investigadores tendem, em geral, a considerar que a finalidade do ensino e aprendizagem de L2 consiste em desenvolver a competência comunicativa e preparar os estudantes para usarem a língua em situações reais de comunicação, o que do ponto de vista da maioria dos investigadores envolve primeiramente conhecimento implícito (R. Ellis, 2008, p. 2).

$\mathrm{Na}$ área da ASL é, assim, possível encontrar várias teorias que adotam posições semelhantes (e.g., DeKeyser, 2007a; R. Ellis, 2005) relativamente à natureza do conhecimento de $L 2$, distinguindo a informação que os estudantes têm sobre a língua de aprendizagem, de um tipo de conhecimento que lhes permite usar essa informação para comunicar, sendo que, de uma perspetiva cognitiva (e.g., R. Ellis et al., 2009; Rebuschat, 2013), uma forma de distinguir o conhecimento de L2 consiste em diferenciar entre conhecimento explícito e implícito.

A primeira diferença entre estes dois tipos de conhecimento consiste, em primeiro lugar, no papel da consciência, sendo que o conhecimento explícito tende a ser descrito como um tipo de informação de que os estudantes têm consciência e que podem verbalizar em termos de regras ou fazendo uma descrição sobre o seu uso, designadamente, com recurso a terminologia metalinguística (Basturkmen, Loewen, \& R. Ellis, 2002; Gutiérrez, 2013), enquanto o conhecimento implícito tem sido descrito como um tipo de conhecimento inconsciente da língua, e não sobre a língua (R. Ellis, 2005, 2009; R. Ellis et al., 2009; Rebuschat, 2013), uma vez que os estudantes não têm, necessariamente, consciência dele e não são capazes de o verbalizar, ou apenas o conseguem descrever em termos de intuições, sendo semelhante ao tipo de conhecimento que os falantes de L1 dispõem para comunicar e que thes permite centrarem-se na mensagem em lugar de se focarem nas formas necessárias para o efeito (Loewen, 2014, p. 21). Por outro lado, e em termos de processamento cognitivo, enquanto o recurso ao conhecimento explícito requer esforço cognitivo e 
tempo necessário para ser recuperado, o que impede uma produção em L2 rápida e sem interrupções, pelo contrário, os estudantes conseguem aceder, rapidamente e de modo inconsciente, ao conhecimento implícito e fazer produções não planeadas.

Não obstante, e ainda que exista, atualmente, algum consenso entre as várias teorias, relativamente ao facto de os estudantes de L2 possuírem dois tipos de conhecimento e de a competência comunicativa envolver primeiramente conhecimento implícito, existem posições teóricas diferentes em relação ao modo como este tipo de conhecimento pode ser desenvolvido (e.g., DeKeyser, 1998; N. Ellis, 1998; R. Ellis, \& Shintani, 2014; Krashen, 2003; Paradis, 2004, 2009; Ullman, 2001, 2004).

Em geral, considera-se (e.g., Krashen, 2003; Macaro \& Masterman, 2006; Rebuschat, 2013) que o conhecimento implícito e o conhecimento explícito se relacionam com a aprendizagem implícita e explícita, e que os dois tipos de aprendizagem constituem processos separados, cujos resultados são armazenados em partes diferentes do cérebro, sendo que a aprendizagem implícita (que ocorre sem intenção ou consciência) resulta em conhecimento implícito, enquanto a aprendizagem explícita (que, geralmente é intencional e evidente) resulta em conhecimento explícito.

Assim, e à semelhança do que se verifica em outras áreas do conhecimento, há poucas dúvidas de que a disponibilização de informação explícita e declarativa sobre L2 pode promover o conhecimento explícito sobre a língua-alvo, no entanto continua a haver alguma controvérsia (e.g., DeKeyser, 2007b; R. Ellis, 2005; Krashen, 2003) quanto à possibilidade de a instrução poder ajudar os estudantes a desenvolver um tipo de conhecimento de L2 que resulte, de facto, no desenvolvimento da competência comunicativa.

A possibilidade de o ensino explícito, por si só, poder resultar na capacidade de os estudantes usarem a língua para comunicar continua, de facto, a ser uma questão polémica, que deu origem à designada "Hipótese de interface", face à qual se distinguem três posições - posição de não interface, posição de interface forte e posição de interface moderada (R. Ellis \& Shintani, 2014, p. 12) - a que se encontram associadas abordagens pedagógicas também diferentes.

A "posição de não interface" (Krashen, 1981, 2003; Paradis, 1994) baseia-se em teorias e investigações de acordo com as quais o conhecimento explícito e o conhecimento implícito envolvem mecanismos diferentes, que se encontram armazenados em partes diferentes do cérebro, aos quais o falante acede mediante processos diferentes (controlado versus automático), e sustenta que o conhecimento explícito não se pode transformar em implícito, por ser neurologicamente distinto. Em termos de implicações pedagógicas, tal significa que o ensino explícito de regras gramaticais resulta, apenas, em conhecimento explícito e que o conhecimento 
implícito se desenvolve, naturalmente, a partir de uma comunicação centrada no sentido (e com ajuda, talvez, de algum foco na forma) ${ }^{10}$, sendo que a melhor opção pedagógica consiste em disponibilizar quantidades significativas de input e envolver os estudantes na comunicação.

Por sua vez, a "posição de interface forte", que é sustentada pela Teoria de Aquisição de Capacidades (e.g., DeKeyser, 1998), considera que o conhecimento implícito decorre do explícito quando este último é automatizado pela prática. Assim, e à semelhança de outras abordagens cognitivas sobre o conhecimento de L2, a Teoria de Aquisição de Capacidades também faz uma distinção entre dois tipos de conhecimento, contudo, em lugar de se referir a eles em termos de conhecimento explícito e implícito, designa-os por conhecimento declarativo e procedimental, propondo três fases no processo de transformação do conhecimento explícito em uma produção fluente em L2: (i) aquisição de conhecimento declarativo; (ii) aquisição de conhecimento procedimental; (iii) e automatização, através da qual os estudantes aprendem a usar o conhecimento procedimental em uma produção fluente e espontânea (Loewen, 2014, p. 27).

Finalmente, é possível encontrar, ainda, autores que defendem uma "posição de interface moderada" (N. Ellis, 2005; R. Ellis, 1993, 1994), de acordo com a qual o conhecimento explícito pode contribuir (indiretamente) para o desenvolvimento do conhecimento implícito, ao promover os processos que se encontram envolvidos na aquisição, em particular, o "registo cognitivo" e o "registo de lapsos" (Schmidt, 1994), o que significa que o conhecimento explícito sobre uma determinada estrutura da língua-alvo permite que os estudantes reconheçam, mais facilmente, determinados aspetos do input e estabeleçam também comparações cognitivas entre o que são capazes de produzir e o que observam no input.

Em suma, existem três perspetivas diferentes sobre a relação entre conhecimento explícito (ou declarativo) e conhecimento implícito (ou procedimental), das quais decorrem abordagens também distintas para o ensino da língua: "a posição de não interface" dá prioridade a abordagens centradas no sentido (como o Ensino de Línguas Baseado em Tarefas e a imersão); a "posição de interface forte" sustenta o modelo de apresentação, prática e produção (ou seja, a ideia de que a estrutura gramatical deve, primeiro, ser apresentada explicitamente e, depois, praticada até ser totalmente

10 Long (1991, pp. 45-46) introduz a expressão "foco na forma" para se referir a um tipo de instrução que promove a atenção acidental para aspetos linguísticos (e.g., gramática, léxico, fonética) durante a atividade comunicativa, isto é, enquanto os estudantes se encontram, primeiramente, centrados no sentido. Em contraste, usa a expressão "foco nas formas" para se referir à instrução de itens linguísticos pré-selecionados, no âmbito de atividades em que a atenção dos estudantes se encontra, primeiramente, centrada na forma e não no sentido. 
procedimentalizada); e, por sua vez, a "posição de interface moderada" sustenta, designadamente, o recurso a técnicas que levem os estudantes a deduzir as suas próprias regras a partir da análise de dados, que também podem ser implementadas no âmbito do Ensino de Línguas Baseado em Tarefas.

\section{Resultados da investigação}

Vários estudos têm analisado os efeitos de diferentes tipos de instrução no desenvolvimento do conhecimento implícito e do explícito, sendo habitual considerar que o ensino explícito resulta em aprendizagem explícita (e.g., Bowles, 2011; Sonbul \& Schmitt, 2013), embora alguns estudos tenham, também, identificado alguns efeitos da instrução explícita no desenvolvimento de conhecimento implícito (R. Ellis, Loewen, \& Erlam 2006; Spada \& Tomita, 2010; White \& Ranta, 2002), ainda que não tenham sido corroborados por outros trabalhos de investigação, como o estudo desenvolvido por Sonbul \& Schmitt (2013), que não aferiu evidências de influência da instrução explícita no conhecimento implícito de certos aspetos do vocabulário.

Assim, e apesar de alguns trabalhos concluírem que a instrução explícita pode ser benéfica para a aquisição de conhecimento implícito, continua a ser necessário desenvolver mais investigação com a finalidade de determinar se estes dados podem ser generalizados (Loewen, 2014, p. 33), mas também identificar variáveis que possam aumentar ou diminuir a eficácia da instrução explícita.

No que diz respeito à relação entre instrução implícita e conhecimento implícito, existem, igualmente, vários estudos que apontam para efeitos positivos: Rebuschat e Williams (2012), por exemplo, investigaram a capacidade de os estudantes adquirirem conhecimento sobre uma língua semiartificial com base apenas na exposição a input, tendo verificado que os estudantes eram capazes de adquirir as regras sintáticas de L2 acidentalmente, usando-as para fazer juízos gramaticais; por seu lado, Li (2010) desenvolveu uma meta-análise, com base em cerca de trinta e sete estudos sobre feedback corretivo ${ }^{11}$ e verificou que, embora o feedback corretivo explícito se tivesse revelado mais eficaz a curto prazo (isto é, em pós-testes imediatos), o feedback implícito era mais eficaz a longo prazo (isto é, em pós-testes mais tardios), levando o autor a concluir que o feedback implícito ${ }^{12}$ contribui para o desenvolvimento do conhecimento implícito, o qual é mais durável, embora leve mais tempo a desenvolver, pelo que os efeitos da instrução implícita podem não ter resultados imediatos.

11 O feedback explícito pode adotar formas diferentes, como correção direta ou explicação metalinguística.

12 No feedback implícito, a correção dos erros produzidos pelo estudante é encoberta (e.g., reformulação). 
Assim, e relativamente à questão de saber de que modo a instrução pode contribuir para o desenvolvimento da competência comunicativa dos estudantes e promover um tipo de conhecimento (implícito) que Ihes permita participar em situações de comunicação espontâneas, embora continue a ser necessário desenvolver mais investigação, alguns dos trabalhos referidos mostram, de facto, que tanto o conhecimento implícito como o conhecimento explícito de L2 podem ser desenvolvidos de várias maneiras, e que a instrução explícita pode ter efeitos positivos no desenvolvimento dos dois tipos de conhecimento, embora a instrução implícita também pareça ser eficaz, pelo que a combinação de instrução implícita e explícita parece ser a melhor opção em sala de aula.

Em muitos contextos educativos, a aprendizagem tende, no entanto, a ser concebida como uma acumulação de conhecimento explícito sobre regras gramaticais e vocabulário, o que, por um lado, dá a noção aos estudantes de que estão a aprender alguma coisa tangível e, por outro, permite, aos professores, aferir o conhecimento (explícito) adquirido pelos estudantes de modo mais objetivo. Contudo, a assunção de que o conhecimento explícito irá permitir, aos estudantes, usar a L2 facilmente para comunicar revela-se problemática, uma vez que tal não acontece necessariamente (Loewen, 2014, p. 25), e implica que a instrução seja planeada de acordo com o que se sabe sobre o processo de aquisição de $L 2^{13}$.

Assim, e embora muitos cursos de línguas continuem a privilegiar o desenvolvimento do conhecimento explícito sobre L2, algumas propostas de ensino atuais, como o Ensino de Línguas Baseado em Tarefas, têm sustentado, pelo contrário, a importância de se criarem condições em sala de aula que promovam, sobretudo, o desenvolvimento do conhecimento implícito e do conhecimento procedimental em que a competência comunicativa se baseia, mas sem descurar o conhecimento explícito e a aprendizagem de aspetos gramaticais (R. Ellis \& Shintani, 2014, p. 23), como iremos abordar na secção seguinte.

\section{0 ensino de línguas baseado em tarefas}

A proposta de programas de ensino de línguas baseados em tarefas decorre, fundamentalmente, do reconhecimento de que não é possível especificar, exatamente, o que o estudante vai aprender, sendo, por isso, necessário abandonar a pré-seleção

13 Segundo R. Ellis (2003, p. 209) a lacuna apontada aos programas linguísticos pode decorrer mais do modo como é implementado (isto é, da metodologia) do que do design. 
de itens linguísticos em que os programas linguísticos ${ }^{14}$ se baseiam e, no seu lugar, especificar o conteúdo em termos de unidades holísticas de comunicação (isto é, tarefas), promovendo-se, assim, um ensino "através da comunicação" em detrimento de "um ensino para a comunicação".

Long e Crookes (1992, pp. 30-31) sustentam que os programas linguísticos partem de um modelo de aquisição de L2 que não é sustentado pela investigação e que se traduz, designadamente, na expetativa de um uso imediato e correto das estruturas ensinadas (muitas vezes, no final de uma única lição), embora sublinhem que os programas nocionais-funcionais ${ }^{15}$ também não permitem ultrapassar este problema, uma vez que uma determinada noção ou função não constitui uma "unidade de aquisição" mais plausível do que uma estrutura gramatical, argumentando que a passagem de um programa estrutural para um nocional-funcional não envolve uma mudança de pensamento significativa, apesar de ter facilitado a conceção de programas baseados nas necessidades dos estudantes (R. Ellis, 2003, p. 207).

A reavaliação de programas linguísticos resulta, assim, em particular, do facto de os estudantes não apresentarem capacidades comunicativas significativas em L2, apesar de terem recebido instrução baseada neste tipo de programas durante vários anos (Stern, 1983), mas também por haver evidências de que os estudantes aprendem de acordo com o seu próprio "programa em construção" e independentemente do que lhes é ensinado (Corder, 1967).

O Ensino de Línguas Baseado em Tarefas (ELBT) preconiza, assim, uma metodologia que pode ser considerada uma extensão da Abordagem Comunicativa (Richards, 2006, p. 27) e que surge, essencialmente, como uma resposta às limitações referidas, baseando-se na assunção de que a instrução tem de ser compatível com o processo natural de aquisição e propondo, em alternativa, que as tarefas constituem uma unidade mais adequada para a especificação das necessidades dos estudantes.

Relativamente à sua planificação, apesar de não haver uma pré-determinação de estruturas, noções ou funções a ensinar no ELBT, alguns autores (e.g., R. Ellis, 2003; Skehan, 1996) consideram que este tipo de ensino envolve, não obstante, a tomada de decisões sobre o conteúdo (as tarefas a incluir no programa) e a metodologia (o modo como as tarefas são usadas em sala de aula).

14 Os programas linguísticos são planificações construídas em torno de unidades linguísticas, em termos estruturais (como uma lista de características gramaticais), ou em termos nocionais/funcionais (R. Ellis, 2003, p. 28).

15 O designado programa nocional-funcional consiste na identificação de noções (e.g., "possibilidade" ou "certeza") e funções (e.g., "pedir informação" ou "desculpar") e corresponde, essencialmente, a um programa linguístico, no qual se identifica o conteúdo linguístico a ensinar, sendo igualmente intervencionista e externo ao estudante. 
Para ilustrar a sua planificação, R. Ellis (2003, p. 206) propõe dois tipos de programas baseados em tarefas: um constituído, inteiramente, por tarefas sem foco ${ }^{16}$ e outro apenas por tarefas com foco ${ }^{17}$. No primeiro caso, a elaboração do programa implica, apenas, selecionar um conjunto de tarefas adequadas ao público-alvo, não sendo necessário especificar o conteúdo linguístico; enquanto a segunda opção dá origem, simultaneamente, a um programa baseado em tarefas e a um programa linguístico (R. Ellis \& Shintani, 2014, p. 140), sendo necessário especificar as tarefas e o conteúdo linguístico.

Uma terceira possibilidade, segundo R. Ellis (2002, pp. 17-34), consiste, ainda, em elaborar um programa constituído por uma conjugação de tarefas com foco e sem foco, introduzindo-se, assim, um foco na forma em um programa centrado no sentido. Neste caso, o programa é constituído por dois módulos separados (um módulo comunicativo e um módulo centrado no código), sem que seja feita qualquer tentativa para integrar o conteúdo e a forma (cabendo aos professores tomar decisões sobre quando devem usar as tarefas do módulo centrado no código) e que permite solucionar alguns dos problemas que se colocam à pré-seleção de conteúdo linguístico: o primeiro módulo (comunicativo) constitui a parte principal do programa e é composto por tarefas sem foco, que oferecem aos estudantes a oportunidade para desenvolverem a fluência, a correção e a complexidade em atividades centradas no sentido ${ }^{18}$, enquanto o segundo módulo (centrado no código) constitui uma componente secundária do programa e destina-se a orientar uma eventual intervenção do professor, relativamente a aspetos que se revelem difíceis de aprender de "forma natural".

R. Ellis (2003, p. 236) propõe também que, em uma conceção modular como esta, a fase inicial de aprendizagem deve ser totalmente reservada ao módulo comunicativo e que o módulo centrado no código poderá ser gradualmente introduzido, à medida que os estudantes vão desenvolvendo a sua competência comunicativa.

Na sua abordagem, o autor sustenta que, na fase inicial de aprendizagem da língua, os erros abundam na produção dos estudantes, não fazendo sentido tentar abordá-los na sua totalidade, uma vez que muitos deles vão sendo naturalmente eliminados, pelo

16 As tarefas sem foco são elaboradas com a finalidade de estimular a compreensão e produção geral, distinguindo-se das tarefas com foco que têm como objetivo promover a atenção acidental para estruturas específicas da língua-alvo.

17 É importante, no entanto, sublinhar que um programa composto por tarefas com foco difere de um programa estrutural, pelo facto de no ELBT os estudantes não terem conhecimento das estruturas em que se devem focar.

18 Tal não significa que os estudantes não dão atenção à forma quando realizam as tarefas do módulo comunicativo, apenas quer dizer que não é feita qualquer tentativa em pré-determinar essas formas. 
que a necessidade de um foco na forma surge mais tarde, quando os estudantes já adquiriram alguma habilidade comunicativa e correm o risco de fossilização, o que significa que o módulo centrado no código é introduzido com a finalidade de chamar a atenção para a forma e destabilizar a interlíngua dos estudantes.

R. Ellis (2003, p. 238) considera, assim, que uma abordagem modular é mais compatível com o processo de aquisição de L2, uma vez que o programa de ensino é constituído por dois módulos independentes (formados por tarefas com foco e sem foco) e possibilita que os estudantes se foquem na forma quando já têm um nível de proficiência que Ihes permite, de facto, beneficiar mais da atenção à forma.

Esta proposta sustenta, portanto, uma inversão na sequência tradicional de instrução, considerando mais adequado começar pelo desenvolvimento de vocabulário e de estratégias destinadas a usar o léxico em contexto, e promover, mais tarde, a atenção dos estudantes para o sistema de regras.

\section{ELBT e o desenvolvimento do conhecimento implícito e explícito}

Uma orientação fundamental, que se deve ter em conta na planificação de cursos de línguas baseados em tarefas e dirigidos a estudantes adultos, diz respeito à necessidade de a instrução promover o conhecimento implícito sem negligenciar o conhecimento explícito (R. Ellis \& Shintani, 2014, p. 23), pois embora a aprendizagem acidental (mediante exposição extensiva a input) possa ser benéfica para a aquisição de L2, para este tipo de estudantes, poderá também ser útil recorrer a tipos de instrução mais intencionais, através de um foco na forma e instrução explícita (Loewen, 2014, p. 109), pois, como vimos, quanto mais avançada a idade, mais o indivíduo recorre à sua memória declarativa para aprender uma L2, ou seja, o aprendente tardio de uma segunda língua recorre a formas de aprendizagem diferentes das que estão ao alcance do indivíduo no momento de aquisição da sua língua materna.

Neste sentido, a instrução deve também desenvolver o conhecimento explícito sobre gramática, vocabulário, fonologia ou pragmática, pois, apesar de não ser claro até que ponto é possível transferir esse conhecimento para o discurso espontâneo, poderá ser útil por outras razões. De facto, como referimos, alguns autores, como R. Ellis (2003, p. 149), sustentam uma "posição de interface moderada", e propõem que, apesar de o conhecimento explícito não contribuir, diretamente, para o desenvolvimento do conhecimento implícito, cria condições para o seu desenvolvimento, ao ajudar os estudantes a registarem cognitivamente formas no input, a registarem 
diferenças (noticing the gap) entre a sua produção e o modo como a estrutura é usada no input e, ainda, a monitorizar a produção.

Assim, e de acordo com a "posição de interface moderada", pensamos também que o ensino de L2 dirigido a estudantes adultos deve promover tanto o desenvolvimento do conhecimento implícito como o conhecimento explícito, apesar de os professores não deverem assumir que o conhecimento explícito pode ser convertido diretamente em conhecimento implícito.

Quanto ao tipo de atividade mais adequado para o efeito, R. Ellis (2003, p. 150) sustenta que, para além de tarefas sem foco, é possível elaborar determinadas tarefas com foco para promover, por um lado, a aquisição de aspetos específicos do conhecimento implícito (mediante as designadas tarefas de produção estruturada, estratégias de enriquecimento de input e tarefas de interpretação) e, por outro, para desenvolver o conhecimento explícito (mediante tarefas de promoção da consciência linguística), mas também para promover a automatização de formas (R. Ellis, p. 261) que os estudantes já tenham começado a usar, mas que ainda não dominam - mediante tarefas de prática/produção mais tradicionais ${ }^{19}$.

A incorporação de tarefas com foco suscita, contudo, a questão da seleção e sequencialização de formas específicas, o que coloca, novamente, o problema da correspondência entre o "programa de ensino" e o "programa em construção" do estudante. Contudo, quando o objetivo é o desenvolvimento do conhecimento implícito, os responsáveis pela elaboração do programa não podem determinar, com antecedência, as formas específicas que irão ser abordadas nem o momento em que devem ser tratadas, sendo apenas possível identificar conteúdo linguístico com potencial para ser integrado em tarefas com foco, elaborando uma lista de verificação composta por itens, que se revelem difíceis de adquirir de modo natural, e de procedimentos destinados a decidir quando um item particular pode ser, utilmente, trabalhado. No entanto, e em particular com estudantes adultos, como referimos, é importante promover também o conhecimento explícito e, neste caso, é possível elaborar um programa graduado, que se pode basear em uma análise do tipo de erros ou dificuldades que os estudantes enfrentam na aprendizagem de L2, pois aquilo que pode ser difícil de adquirir implicitamente poderá ser fácil de compreender declarativamente, pelo que o problema de aprendizagem que impossibilita a sequencialização em relação ao conhecimento implícito não se coloca em relação ao conhecimento explícito.

19 R. Ellis (2003), ao contrário de outros autores, considera que o ELBT é compatível, em alguns aspetos, com metodologias mais tradicionais, designadamente, na fase pós-tarefa, para promover a automatização de aspetos que os estudantes já adquiriram, mas ainda não controlam totalmente. 


\section{Conclusões}

Os vários trabalhos de investigação referidos permitem concluir que existe, atualmente, reconhecimento de que para desenvolver a competência comunicativa e para que os estudantes adultos possam atingir níveis elevados de fluência e correção é importante recorrer a abordagens que combinem tipos de comunicação centrados no sentido e na forma (Loewen, 2014, p. 54), o que pode ser promovido, adotando, em sala de aula, tipos de instrução implícitos e explícitos.

Verificámos também que o ELBT pode constituir uma alternativa para o ensino de L2, sendo, atualmente, considerada uma abordagem capaz de promover as condições necessárias ao desenvolvimento da competência comunicativa (R. Ellis \& Shintani, 2014; Littlewood, 2011; Mackey, 2007, 2012; Skehan, Bei, Qian, \& Wang, 2012; Van den Braden, Bygate, \& Norris, 2009), sem descurar o desenvolvimento gramatical (Carless, 2012) e outras componentes da competência comunicativa (Shehadeh, 2012).

Concluímos, em suma, que, embora os investigadores na área da ASL não estejam de acordo em relação ao modo como a instrução pode contribuir para o desenvolvimento de L2, o ELBT e a instrução com foco na forma podem constituir abordagens pedagógicas adequadas para promover o tipo de processo de aprendizagem que se considera facilitar a aquisição de L2 (Mackey, 2012, p. 57), ou seja, que o recurso a tarefas - isto é, atividades pedagógicas que requerem um uso pragmático da língua, com o objetivo global de promover o desenvolvimento de L2 (Bygate \& Samuda, 2009, p. 93) - pode potenciar o tipo de interação e um foco em elementos particulares da língua que são considerados benéficos para a aquisição, pelo que deveria ser uma abordagem mais divulgada junto dos professores de línguas e dos responsáveis pela elaboração de materiais didáticos dirigidos à aprendizagem de L2.

\section{Referências bibliográficas}

Basturkmen, H., Loewen, S., \& Ellis, R. (2002). Metalanguage in focus on form in the communicative classroom. Language Awareness, 11, 1-13.

Beretta, A., \& Davies, A. (1985). Evaluation of the Bangalore project. ELT Journal, 39, 121-127.

Bowles, M. (2011). Measuring implicit and explicit linguistic knowledge: What can heritage language learners contribute? Studies in Second Language Acquisition, 33, 247-271.

Bygate, M., \& Samuda, V. (2009). Creating pressure in task pedagogy. In A. Mackey \& C. Polio (Eds.), Multiple perspectives on interaction (pp. 90-116). New York and London: Routledge. 
Carless, D. (2012). TBLT in EFL settings: Looking back and moving forward. In A. Shehadeh \& C. A. Coombe (Eds.), Task-based language teaching in language contexts: Research and implementation (pp. 346-358). Amsterdam: John Benjamins Publishing Company.

Corder, S. P. (1967). The significance of learners' errors. International Review of Applied Linguistics, 5, 161-169.

DeKeyser, R. (1998). Beyond focus on form: Cognitive perspectives on learning and practicing second language grammar. In C. Doughty \& J. Williams (Eds.), Focus on form in classroom second language acquisition (pp. 42-46). Cambridge: Cambridge University Press.

DeKeyser, R. (2007a). Skill acquisition theory. In J. Williams \& B. VanPatten (Eds.), Theories in second language acquisition: An introduction (pp. 97-113). Mahwah: Erlbaum.

DeKeyser, R. (2007b). The future of practice. In R. DeKeyser (Ed.), Practicing in a second language: Perspectives from applied linguistics and cognitive psychology (pp. 287-304). New York: Cambridge University Press.

De la Fuente, M. J. (2006). Classroom L2 vocabulary acquisition: Investigating the role of pedagogical tasks and form-focused instruction. Language Teaching Research, 10(3), 263-295. doi:10.1191/1362168806/r196oa

Ellis, N. (1998). Emergentism, connectionism and language learning. Language Learning, 48, 631-664.

Ellis, N. (2005). At the interface: Dynamic, interactions of explicit and implicit knowledge. Studies in Second Language Acquisition, 27(2), 305-352.

Ellis, N. (2007). The associative-cognitive creed. In B. Van Patten \& J. Williams (Eds.), Theories in second language acquisition: An introduction (pp. 77-96). Mahwah, NJ: Lawrence Erlbaum Associates.

Ellis, R. (1993). Second language acquisition and the structural syllabus. TESOL Quarterly, 27, 91-113.

Ellis, R. (1994). The study of second language acquisition. Oxford: Oxford University Press.

Ellis, R. (2002). The place of grammar instruction in the second/foreign language curriculum. In E. Hinkel \& S. Fotos (Eds.), New perspectives on grammar teaching in second language classrooms (pp. 17-34). London: Routledge.

Ellis, R. (2003). Task-based language learning and teaching. Oxford: Oxford University Press.

Ellis, R. (2005). Principles of instructed language learning. Asian EFL Journal, 7(3), 9-24.

Ellis, R. (2008). Principles of instructed second language acquisition. CALdigest, 1-6. Consultado em https://moodle2.unifr.ch/pluginfile.php/166786/mod_resource/content/2/ Texte/03d_Ellis_2008_Instructed2ndLangFinalWeb.pdf

Ellis, R. (2009). Implicit and explicit learning, knowledge, and instruction. In R. Ellis, S. Loewen, C. Elder, J. Philp, H. Reinders, \& R. Erlam (Eds.), Implicit and explicit knowledge in second language learning, testing and teaching (pp. 3-25). Bristol: Multilingual Matters.

Ellis, R., Loewen, S., Elder, C., Erlam, R., Philp, J., \& Reinders, H. (2009). Implicit and explicit knowledge in second language learning, testing and teaching. Bristol: Multilingual Matters. 
Ellis, R., Loewen, S., \& Erlam, R. (2006). Implicit and explicit corrective feedback and the acquisition of L2 grammar. Studies in Second Language Acquisition, 28, 339-368.

Ellis, R., \& Shintani, N. (2014). Exploring language pedagogy through second language acquisition. London: Routledge.

Ellis, R., Tanaka, Y., \& Yamazaki, A. (1994). Classroom interaction, comprehension, and the acquisition of $L 2$ word meanings. Language Learning, 44, 449-491.

Flores, C. (2013). Português língua não materna. Discutindo conceitos de uma perspetiva linguística. In R. Bizarro, M. Moreira, \& C. Flores (Orgs.), Português língua não materna: Investigação e ensino (pp. 35-46). Lisboa: Lidel.

Gass, S., \& Selinker, L. (2008). Second language acquisition: An introductory course. London: Lawrence Erlbaum.

Gutiérrez, X. (2013). Metalinguistic knowledge, metalingual knowledge and proficiency in L2 Spanish. Language Awareness, 22(2), 176-191.

Housen, A., \& Pierrard, M. (2005). Investigations in instructed second language acquisition. Berlin: Mouton de Gruyter.

Howard, M. (2005). Second language acquisition in a study abroad context: A comparative investigation of the effects of study abroad and foreign language instruction on the L2 learner's grammatical development. In A. Housen \& M. Pierrard (Eds.), Investigations in instructed second language acquisition (pp. 495-530). Berlin: Mouton de Gruyter.

Krashen, S. (1981). Second language acquisition and second language learning. Oxford: Pergamon.

Krashen, S. (1982). Principles and practice in second language acquisition. Oxford: Pergamon.

Krashen, S. (1985). The input hypothesis: Issues and implications. London: Longman.

Krashen, S. (2003). Explorations in language acquisition and use: The Taipei lectures. Portsmouth: Heinemann.

Lantolf, J. P., \& Thorne, S. (2007). Sociocultural theory and second language learning. In B. Van Patten \& J. Williams (Eds.), Theories in second language acquisition: An introduction (pp. 201-224). Mahwah: Lawrence Erlbaum Associates.

Li, S. (2010). The effectiveness of corrective feedback in SLA: A meta-analysis. Language Learning, 60, 309-365.

Littlewood, W. (2011). Communicative language teaching: An expanding concept for a changing world. In E. Hinkel (Ed.), Handbook of research in second language teaching and learning (pp. 541-57). New York: Routledge.

Loewen, S. (2014). Introduction to instructed second language acquisition London: Routledge.

Long, M. (1983). Does second language instruction make a difference? A review of the research. TESOL Quarterly, 17, 359-382.

Long, M. (1991). Focus on form: A design feature in language teaching methodology. In K. De Bot, R. Ginsberg, \& C. Kramsch (Eds.), Foreign language research in cross-cultural perspective (pp. 39-52). Amsterdam: John Benjamins Publishing Company.

Long, M., \& Crookes, G. (1992). Three approaches to task-based syllabus design. TESOL Quarterly, 26, 27-56.

Macaro, E., \& Masterman, L. (2006). Does intensive explicit grammar instruction make all the difference? Language Teaching Research, 10, 297-327. 
Mackey, A. (1999). Input, interaction and second language development. Studies in Second Language Acquisition, 21, 557-587.

Mackey, A. (2007). Conversation interaction in second language acquisition: A series of empirical studies. Oxford: Oxford University Press.

Mackey, A. (2012). Input, interaction and corrective feedback in L2 learning. Oxford: Oxford University Press.

Mackey, A., \& Goo, J. (2007). Interaction research in SLA: A meta-analysis and research synthesis. In A. Mackey (Ed.), Conversational interaction in second language acquisition (pp. 407-452). Oxford: Oxford University Press.

Norris, J., \& Ortega, L. (2000). Effectiveness of L2 instruction: A research synthesis and quantitative meta-analysis. Language Learning, 50, 417-528.

Ortega, L. (2007). Second language learning explained? SLA across nine contemporary theories. In B. Van Patten \& J. Williams (Eds.), Theories in second language acquisition: An introduction (pp. 221-246). Mahwah: Lawrence Erlbaum Associates.

Paradis, M. (1994). Neurolinguistic aspects of implicit and explicit memory: Implications for bilingualism. In N. Ellis (Ed.), Implicit and explicit learning of second languages (pp. 393-419). London: Academic Press.

Paradis, M. (2004). A neurolinguistic theory of bilingualism. Amsterdam: John Benjamins Publishing Company.

Paradis, M. (2009). Declarative and procedural determinants of second languages. Amsterdam: John Benjamins Publishing Company.

Prabhu, N. S. (1987). Second language pedagogy. Oxford: Oxford University Press.

Rebuschat, P. (2013). Measuring implicit and explicit knowledge in second language acquisition. Language Learning, 63, 595-626.

Rebuschat, P., \& Williams, J. (2012). Implicit and explicit knowledge in second language acquisition. Applied Psycholinguistics, 33, 829-856.

Richards, J. C. (2006). Communicative language teaching today. New York: Cambridge University Press.

Schmidt, R. (1994). Deconstructing consciousness in search of useful definitions for applied linguistics. AlLA Review, 11, 11-26.

Shehadeh, A. (2012). Broadening the perspective of task-based language teaching scholarship: The contribution of research in foreign language contexts. In A. Shehadeh \& C. Coombe (Eds.), Task-based language teaching in foreign language contexts: Research and implementation (pp. 1-20). Amsterdam: John Benjamins Publishing Company.

Shintani, N. (2013). The effect of focus on form and focus on forms instruction on the acquisition of productive knowledge of $\mathrm{L} 2$ vocabulary by young beginning-level learners. TESOL Quarterly, 47(1), 36-62.

Shintani, N., Li, S., \& Ellis, R. (2013). Comprehension-based versus production-based grammar instruction: A meta-analysis of comparative studies. Language Learning, 63, 296-329.

Skehan, P. (1996). A framework for the implementation of task-based instruction. Applied Linguistics, 17, 38-62.

Skehan, P., Bei, X, Qian, L., \& Wang, Z. (2012). The task is not enough: Processing approaches to task-based performance. Language Teaching Research, 16, 170-187. 
Sonbul, S., \& Schmitt, N. (2013). Explicit and implicit lexical knowledge: Acquisition of colocations under different input conditions. Language Learning, 63, 121-159.

Spada, N., \& Tomita, Y. (2010). Interactions between type of instruction and type of language feature: A meta-analysis. Language Learning, 60(2), 1-46.

Stern, H. (1983). Fundamental concepts of language teaching. Oxford: Oxford University Press.

Ullman, M. T. (2001). A Neurocognitive perspective on language: The declarative/procedural model. Nature Reviews Neuroscience, 2, 717-726.

Ullman, M. T. (2004). Contributions of memory circuits to language: The declarative/procedural model. Cognition, 92, 231-270.

Van den Braden, K., Bygate, M., \& Norris, J. (2009). Task-based language teaching: A reader. Amsterdam: John Benjamins Publishing Company.

Vygotsky, L. S. (1962). Thought and language. Cambridge: MIT Press.

Vygotsky, L. S. (1978). Mind in society: The development of higher psychological processes. Cambridge: Harvard University Press.

White, J., \& Ranta, L. (2002). Examining the interface between metalinguistic knowledge and oral production in a second language. Language Awareness, 11, 259-290. 